\title{
GCU
}

Glasgow Caledonian

University

University for the Common Good

\section{Uptake of hepatitis C specialist services and treatment following diagnosis by dried blood spot in Scotland}

McAllister, Georgina; Innes, Hamish; Mcleod, Allan; Dillon, John F.; Hayes, Peter C.; Fox, Ray; Barclay, Stephen T.; Templeton, Kate; Aitken, Celia; Gunson, Rory; Goldberg, David; Hutchinson, Sharon J.

Published in:

Journal of Clinical Virology

DOI:

10.1016/j.jcv.2014.09.004

Publication date:

2014

Document Version

Author accepted manuscript

Link to publication in ResearchOnline

Citation for published version (Harvard):

McAllister, G, Innes, H, Mcleod, A, Dillon, JF, Hayes, PC, Fox, R, Barclay, ST, Templeton, K, Aitken, C, Gunson, R, Goldberg, D \& Hutchinson, SJ 2014, 'Uptake of hepatitis C specialist services and treatment following diagnosis by dried blood spot in Scotland', Journal of Clinical Virology, vol. 61, no. 3, pp. 359-364. https://doi.org/10.1016/j.jcv.2014.09.004

\section{General rights}

Copyright and moral rights for the publications made accessible in the public portal are retained by the authors and/or other copyright owners and it is a condition of accessing publications that users recognise and abide by the legal requirements associated with these rights.

Take down policy

If you believe that this document breaches copyright please view our takedown policy at https://edshare.gcu.ac.uk/id/eprint/5179 for details of how to contact us. 


\section{Background}

4 Dried blood spot (DBS) testing for hepatitis C (HCV) was introduced to Scotland in

5 2009. This minimally invasive specimen provides an alternative to venipuncture and

6 can overcome barriers to testing in people who inject drugs (PWID).

\section{Objectives}

8 The objective of this study was to determine rates and predictors of: exposure to

$9 \mathrm{HCV}$, attendance at specialist clinics and anti-viral treatment initiation among the

10 DBS tested population in Scotland.

\section{Study design}

12 DBS testing records were deterministically linked to the Scottish HCV Clinical

13 database prior to logistic regression analysis.

\section{Results}

15 In the first two years of usage in Scotland, 1322 individuals were tested by DBS of

16 which 476 were found to have an active HCV infection. Linkage analysis showed that

$1732 \%$ had attended a specialist clinic within 12 months of their specimen collection

18 date and $18 \%$ had begun anti-viral therapy within 18 months of their specimen

19 collection date. A significantly reduced likelihood of attendance at a specialist clinic

20 was evident amongst younger individuals ( $<35$ years $)$, those of unknown ethnic origin

21 and those not reporting injecting drug use as a risk factor.

\section{Conclusion}

23 We conclude that DBS testing in non-clinical settings has the potential to increase

24 diagnosis and, with sufficient support, treatment of HCV infection among PWID. 


\section{Background}

\section{(1)}

In Scotland, $0.8 \%$ of the population aged 15-59 years had been diagnosed with hepatitis C virus (HCV) antibodies by the end of 2012 [1]. The majority of these infections occur in individuals with a history of injecting drug use [2] and recent estimates suggest that around half of people infected with HCV remain undiagnosed [1]. To tackle the epidemic of HCV in Scotland, the Hepatitis C Action Plan for Scotland was launched in September 2006 [3]. In its initial Phase (September 2006 March 2008) the Action Plan identified poor venous access amongst people who inject drugs (PWID), along with a shortage of trained phlebotomists, and the long interval between testing and return of results, as barriers to testing and diagnosis of HCV in this population [4]. Dried blood spots (DBS), drops of whole blood from a finger prick dried onto filter paper, provide an alternative to whole blood specimens collected by venipuncture and can overcome the majority of barriers to HCV testing outlined above $[5,6,7,8]$. As a result of the Action Plan, DBS testing for HCV diagnosis was introduced in Scotland in May 2009. Now that DBS testing is well established in Scotland, the outcomes of DBS testing are quantifiable to give a better understanding of the utility of the DBS approach.

\section{Objectives}

\section{6}

7 The objective of this study was to determine the proportion of those tested by DBS in Scotland who had been exposed to $\mathrm{HCV}$; of those diagnosed as being currently infected with $\mathrm{HCV}$ the proportion attending a specialist clinic and, of those, the proportion who were initiated on anti-viral treatment. Epidemiological information 
51 collected alongside the DBS specimens is also analysed to identify predictors of 52 exposure, attendance and treatment initiation amongst this population. 


\section{Study Design}

\section{Data Sources and Linkage}

The Scottish Hepatitis C Clinical Database, held at Health Protection Scotland (HPS), contains clinical follow-up data for $\mathrm{HCV}$-infected patients attending 17 specialist clinics across Scotland. These data include attendance dates, treatment episodes, demographic, clinical, virological, and patient identifiers (date of birth, sex, surname Soundex (a consonant-only phonetic encoding), and forename initial). Data were restricted to individuals on the database on 31 December 2012 and at this date the database contained records for 14,298 individuals with sufficient identifiers for linkage.

HPS also maintains records on all DBS testing in Scotland since May 2009. The DBS database contains information on dates and result(s) of $\mathrm{HCV}$ antibody and reverse transcriptase polymerase chain reaction (RT-PCR) testing, source, ethnicity, risk activitie(s), length of injecting career and limited identifying information (i.e., date of birth, sex, surname Soundex and forename initial). On 31 December 2010 this database comprised records for 1448 specimens relating to 1322 individuals.

Records from the DBS database (up to 31 December 2010) were deterministically linked to individuals on the HCV Clinical database (to 31 December 2012); a complete match on surname Soundex, gender, DOB, and first initial was required for a successful link. 
Three main outcomes were analysed: (a) anti-HCV positivity amongst all individuals tested by DBS for HCV since the inception of the DBS testing programme in Scotland (May 2009) to 31 December 2010, (b) first clinic attendance amongst all chronically $\mathrm{HCV}$-infected persons recorded as being tested by DBS for HCV infection between May 2009 and 31 December 2010 and (c) initiation on antiviral therapy amongst the chronically $\mathrm{HCV}$-infected patients attending a specialist clinic. Univariate and multivariate logistic regression modelling was used to examine the association between the covariates sex, age at diagnosis (grouped into $<35$ years, $\geq 35$ years), ethnicity (White, Unknown/Non-white), Source of DBS (Community Addiction Team/Harm Reduction, Other) and time since onset of injecting $(\leq 10$ years, $>10$ years, Not Known (PWID), Non-PWID) and the outcomes: 'HCV antibody positive' (Table 1), 'first clinic attendance within 12 months of diagnosis by DBS' (Table 2) and

91 'initiation on antiviral therapy within 18 months of DBS specimen collection' (Table 3). For the latter analysis the variable 'Risk Factor' (Current PWID, Past PWID, Non-PWID/Unknown) was also included. For the Risk Factor variable data collected on length of injecting career (including age of first and last injection) was used, where available, to categorise individuals as past PWID and present PWID, with any individual giving a date of last injecting drug use as five or more years prior to the DBS specimen collection date classified as a past PWID.

98

99 All analysis was carried out in R 3.0.1 [8]. Exact p-values are provided except where 100 $\mathrm{P}<0.001$. 
103 In 2009/10 DBS specimens were collected from 1322 individuals in Scotland for 104 HCV screening. Of these individuals 55\% $(n=728)$ were seropositive for antibody to $105 \mathrm{HCV}$, and approximately two-thirds $(65.4 \%(\mathrm{n}=476))$ had an active HCV infection

106 (Figure 1). Table 1 presents characteristics of the overall study sample, according to 107 HCV antibody prevalence. The majority (70\%) were males, although HCV antibody 108 prevalence in both sexes was equal at 55\%. The average age of all DBS tested 109 individuals was 36 , with $45 \%$ of individuals falling into the $<35 \mathrm{yrs}$ age category and $11055 \%$ into the $\geq 35 \mathrm{yrs}$ category. Antibody prevalence was significantly higher in the 111 older age category compared to the younger; 64\% (95\% CI: $60-67 \%$ ) and $45 \%$ (95\%

112 CI: $41-49 \%)$ respectively. White was the main ethnicity $(82.8 \%)$, the remainder 113 being of unknown (16.5\%) or non-white (0.7\%) ethnicity. Most individuals $(89.3 \%)$ 114 were tested in a community addiction team or harm reduction setting as opposed to 115 other settings (hospital (3.8\%), GP $(1.7 \%)$, prison $(0.6 \%)$ or private $(4.6 \%)$ ).

\section{Odds of HCV antibody}

118 Multifactorial logistic regression analysis found age to be related to odds of antibody

119 positivity, with those aged $\geq 35$ years significantly more likely ( $A O R=1.93,95 \%$

120 CI:1.51 - 2.47) than those aged $<35$ years to be antibody positive. The adjusted odds

121 ratio of ethnicity was also positively associated with prevalence. Individuals who

122 were recorded as being of white ethnic origin being more likely ( $\mathrm{AOR}=2.00,95 \% \mathrm{CI}$ :

$1231.42-2.85)$ to be antibody positive as those of unknown/non-white ethnic origin. 
125 PWID are well known to be at increased risk of infection with hepatitis C, particularly 126 those with longer injecting histories. The majority of individuals (85.6\%) tested by

127 DBS reported being/having been a PWID; those who did not report injecting drug use 128 as a risk factor were less likely to be antibody positive (AOR=0.28, 95\% CI: 0.17 129 0.39) than those who had commenced injecting in the previous ten years. There was a 130 marked increase in prevalence between individuals who had injected for 10 years or 131 less $(46.8 \%)$ and individuals with injecting histories of over 10 years $(80.0 \%)$. This 132 translated into a 3.6-fold increased odds of $\mathrm{HCV}$ exposure for the individuals with 133 injecting histories of over a decade (AOR=3.58, 95\% CI: $2.36-5.45)$ in the adjusted 134 analysis. Finally, although not significant in the multifactorial analysis, individuals 135 tested in a community addiction clinic/harm reduction setting $(\mathrm{n}=1180)$ were more 136 likely $(\mathrm{OR}=1.84 .95 \% \mathrm{CI}: 1.30-2.63)$ to be positive for antibody to $\mathrm{HCV}$ as those 137 tested in other settings in the univariate analysis (Table 1).

139 Attendance at Specialist Hepatitis Clinics within 12 months of DBS specimen.

140 Of the 728 individuals known to be antibody positive there were $476(65.4 \%)$ 141 individuals with an active HCV infection as confirmed by RT-PCR. Linkage of these 142 individuals to the Hepatitis C Clinical Database showed that 202 (42.4\%) had ever 143 attended a specialist hepatitis clinic, and 31.9\% (n=152) within 12 months following 144 collection of their DBS specimen (Figure 1). For $7.8 \%(n=37)$ of individuals a date of 145 attendance prior to the DBS specimen date was also found.

147 Univariate analysis did not show any significant relationship between the likelihood 148 of attendance at a specialist hepatitis clinic within the twelve months following 149 diagnosis by DBS and any of the examined variables. However, multifactorial 
logistic regression found a significant relationship between age, risk factor status and

151 ethnicity and attendance at a specialist clinic within 12 months. Individuals aged 35

152 or older were more likely $(\mathrm{AOR}=1.49,95 \% \mathrm{CI}: 1.05-2.13)$ than those aged $<35$ years

153 to attend a treatment clinic within 12 months of DBS diagnosis. Individuals who were

154 recorded as being of a white ethnic background were also more likely ( $\mathrm{AOR}=2.85$,

$15595 \%$ CI: $1.57-5.58)$ to attend a clinic within 12 months as those of a unknown/non-

156 white ethnic background, and there was also a significantly reduced likelihood

$157(\mathrm{AOR}=0.32,95 \% \mathrm{CI}: 0.13-0.71)$ of attendance at a clinic within 12 months for

158 individuals with a non-PWID risk factor (Table 2).

159

160 Initiation on anti-viral therapy within 18 months of DBS specimen date

161 Of the 202 individuals recorded as attending a specialist hepatitis clinic following

162 collection of a DBS specimen in 2009/10, 66 individuals (32.7\%) were recorded

163 beginning anti-viral therapy up to the end of 2012 . For $18.3 \%(n=37)$ of individuals

164 anti-viral therapy was commenced within 18 months of having the DBS specimen

165 collected (Figure 1). Following logistic regression analysis there was no significant

166 association with the likelihood of receiving treatment within 18 months post DBS

167 testing and any of the variables examined in this analysis (Table 3). 
170 Previous studies have demonstrated the effectiveness of DBS in terms of test uptake

171 amongst PWID [5,6,7,8]. To our knowledge, this is the first study to report on the

172 performance of DBS testing in terms of attendance at specialist clinics and treatment

173 initiation. Overall, we found that of the 476 individuals with active HCV infection,

174 tested by DBS in 2009 and 2010, 31.9\% had attended a specialist clinic within 12

175 months of their specimen collection date and, of these, $18.3 \%$ had begun anti-viral

176 therapy within 18 months of their specimen collection date.

177

178 To understand how these figures compare to overall HCV diagnosis in Scotland we 179 can relate our findings to a recent analysis which reviewed similar outcomes, across 180 an overlapping time period, in all new HCV diagnoses in Scotland from 1996 181 onwards. The authors report that, of the 1364 individuals newly diagnosed with 182 chronic HCV in Phase II of the Scottish Hepatitis C Action Plan (1 May 2008 to 31

183 December 2010), 44.5\% attended a specialist hepatitis clinic within 12 months of 184 being diagnosed and $32 \%$ were initiated on anti-viral treatment within the 12 month 185 period following first clinic attendance [10]. Comparing these figures shows that 186 attendance at specialist hepatitis clinics is lower in the DBS tested population at the 18712 month follow-up point (31.9\%) and, although not directly comparable, there also 188 appear to be lower rates of initiation onto anti-viral therapy in the DBS tested 189 population. The populations are not entirely analogous, most notable is that the 190 McDonald et al (2013) study included only new HCV diagnoses whereas this analysis 191 included all diagnoses; among whom there was evidence of prior engagement with 192 specialist services (Figure 1). Since prior knowledge of HCV status may influence 
193 the probability of attendance and treatment this may account for some of the variation 194 between the studies. Finally, in our population, of those chronically infected with $195 \mathrm{HCV}, 95.4 \%$ reported having been/being a PWID and 92.6\% were tested at a

196 drug/counselling clinic, compared to $41.9 \%$ and $9.7 \%$ of the newly diagnosed

197 population. Thus the DBS diagnosed population may well represent a more chaotic 198 group of individuals, involving those who continue to use and inject drugs, which 199 would help to explain the poorer attendance and treatment outcomes amongst this 200 population. Treatment of current PWIDs is still considered problematic by some 201 medical professionals due to concerns over adherence to treatment regimes, medical 202 and psychiatric co-morbidities, psychosocial issues and risk of re-infection [11]. 203 However, there is growing evidence to show that, given adequate support, good 204 treatment outcomes can be achieved among people who continue to inject drugs $205[12,13]$.

207 Looking within our DBS-tested population, logistic regression analysis showed that 208 attendance at specialist hepatitis clinics within 12 months of the DBS specimen 209 collection date was significantly reduced amongst individuals aged less than 35 years 210 and those of unknown/non-white ethnic origin. The significance of the latter finding 211 is unclear as the majority ( $>98 \%$ ) of individuals in this category were of unknown 212 ethnicity. We also found that those in the non-PWID risk factor category are 213 significantly less likely to attend a clinic within 12 months of their DBS collection 214 date, despite being chronically infected with HCV. The basis of this difference is 215 unclear but may reflect the high proportion of PWID in our study and the emphasis of 216 this risk factor amongst healthcare professionals working in DBS testing settings. 217 Awareness of these demographic trends amongst healthcare professionals may enable 
targeted post-test discussion. This analysis did not find any significant association

219 between the variables examined and the likelihood of treatment initiation which may

220 be due to the small sample size and, additionally, our analysis did not have the scope

221 to include the physical, psychological and social factors involved in the decision to 222 treat individuals, and/or willingness to undergo treatment, which have been found to 223 be significant in other studies $[14,15,16]$.

DBS testing was recently estimated to be cost-effective in addiction services settings in the UK at an estimated $£ 14,600$ per quality adjusted life year (QALY) gained [17]. The model was based on $35 \%$ of PWID being successfully referred from testing services to secondary care and 5.5\% of referred PWID being treated within 2 years. The latter variable was based on the assumption that $1 \%$ of infected PWID are treated within 2 years, or $5.5 \%$ of those who attended referral. The authors note that the treatment parameter was a critical factor in assessing the cost-effectiveness of DBS

232 testing since higher treatment rates prevent disease transmission thereby increasing 233 the cost-effectiveness of case-finding interventions. Whilst referral rates in our study 234 are similar to those estimated in the model, we have found a much higher proportion 235 of individuals in secondary care being treated; up to a third within 4 years of their 236 DBS specimen and $18 \%$ within 18 months of their DBS specimen. Although a 237 proportion of our sample were determined to be past-PWID, for whom treatment rates 238 are higher, $86.2 \%$ of the PWID with an active HCV infection had injected within the 239 past five years. As such these findings have great bearing on the cost-effectiveness of 240 DBS testing which was estimated to drop to $£ 4500$ per QALY if $50 \%$ of referred 241 PWID initiated treatment within 2 years [17]. 
243 Our findings are further evidence of the utility of DBS testing in reaching the 244 populations most at risk from $\mathrm{HCV}$ infection and engaging them with specialist 245 hepatitis services. Recent advances in HCV treatment, with the introduction of triple 246 therapy as a standard treatment regime, has significantly improved the rates of 247 sustained virological response [18] and the prospect of interferon-free treatment 248 regimens makes the possibility of an all-oral therapy for HCV conceivable $[19,20]$. 249 Such advances will make treatment a more tolerable therapy and also open the 250 possibility of treatment in the community setting; both of which may facilitate greater 251 uptake in the DBS-tested population in the future. In anticipation of these changes in 252 HCV therapy, and the accompanying possibilities for treatment expansion, the use of 253 DBS should be supported and expanded to maximise engagement with this 254 population. 


\section{Acknowledgements}

256 This work was funded by The Scottish Government as part of the Hepatitis C Action

257 Plan for Scotland. We would also like to thank Stewart Robinson and Hazel Paterson

258 for their assistance with setting up and maintaining the databases. 
260 Funding: This work was funded by The Scottish Government as part of the Hepatitis

261 C Action Plan for Scotland.

263 Competing interests: Peter Hayes has received payment from Gilead, MSD and 264 Jannsen and Roche

266 Ethical approval: Epidemiological data is collected on the laboratory request form and 267 returned along with the dried blood spot specimen to the testing laboratories. All data 268 is handled in accordance to local NHS governance regulations. DBS specimens are 269 always collected with informed consent and the patient is under no obligation to 270 supply any further information along with the specimen. Patients are made aware that 271 any epidemiological information they do provide is held as anonymous surveillance 272 data and will be used for auditing, public health monitoring etc. 


\section{References}

1. Health Protection Agency. HCV in the UK: 2013 Report. London: Health Protection Agency; 2013. Available from [accessed 07/01/2014]: http://www.hpa.org.uk/webc/HPAwebFile/HPAweb_C/1317139502302.

2. McDonald SA, Hutchinson SJ, Schnier C, McLeod A and Goldberg DJ. Estimating the number of injecting drug users in Scotland's HCV-diagnosed population using capture - recapture methods. Epidemiol Infect. 2013;142(1):200-7.

3. Scottish Executive Health Department (SEHD). Hepatitis C Action Plan for Scotland. Phase 1: September2006-August 2008. Edinburgh: Scottish Executive; 2006.

Available

from

[accessed 13/12/2013]: http://www.scotland.gov.uk/Publications/2006/09/150936260.pdf

4. Scottish Executive Health Department (SEHD). Hepatitis C Action Plan for Scotland. Phase II: May 2008 - March2011. Edinburgh: Scottish Executive;

$$
\text { 2008. Available from [accessed 13/12/2013]: }
$$
http://www.scotland.gov.uk/Resource/Doc/222750/0059978.pdf

5. Abdou-Saleh M, Davis P, Rice P, Checinski K, Drummond C, Maxwell D et al. The effectiveness of behavioural interventions in the primary prevention of Hepatitis C amongst injecting drug users: a randomised controlled trial and 
lessons learned. Harm Reduction Journal 2008; 5:25. doi:10.1186/1477-7517-525.

6. Hickman M, McDonald T, Judd A, Nichols T, Hope V, Skidmore S et al. Increasing the uptake of hepatitis $\mathrm{C}$ virus testing among injecting drug users in specialist drug treatment and prison settings by using dried blood spots for diagnostic testing: a cluster randomized controlled trial. J of Viral Hepatitis 2008;15(4):250-254.

7. Craine N, Parry J, O’Toole J, D'Arcy S and Lyons M. Improving blood-borne viral diagnostics: clinical audit of the uptake of dried blood spot testing offered by a substance misuse service. J of Viral Hepatitis 2009;16(3):219-222. doi:10.1111/j.1365-2893.2008.01061.X

8. Jones L, Bates G, McCoy E, Benynon C, MvVeigh J and Bellis M. A systematic review of the effectiveness \& cost-effectiveness of interventions aimed at raising awareness and engaging with groups who are at an increased risk of hepatitis B and C infection. Centre for Public Health, Liverpool John Moores University. 2012. Available from [accessed 03/12/2013]: http://www.nice.org.uk/nicemedia/live/11957/5946/5946.pdf

9. R Core Team. R: A language and environment for statistical computing. R Foundation for Statistical Computing, Vienna, Austria. [Internet]. 2013 Available from[accessed 03/12/2013]: http://www.R-project.org/ 
10. McDonald SA, Hutchinson SJ, Innes HA, Allen S, Bramley P, Bhattacharyya D et al. Increased attendance at specialist hepatitis clinics and initiation of antiviral treatment among persons chronically infected with hepatitis C: Examining the early impact of Scotland's Hepatitis C Action Plan. J Viral Hep. 2013. doi:10.1111/jvh.12153.

11. Edlin BR, Kresina TF, Raymond DB, Carden MR, Gourevitch MN, Rich JD et al. Overcoming barriers to prevention, care, and treatment of hepatitis $\mathrm{C}$ in illicit drug users. Clin Infect Dis. 2005 Apr 15;40(Suppl 5):S276-85.

12. Aspinall EJ, Corson S, Doyle JS, Grebely J, Hutchinson SJ, Dore GJ et al. Treatment of Hepatitis C Virus Infection Among People Who Are Actively Injecting Drugs: A Systematic Review and Meta-analysis Clin Infect Dis. 2013;57(Suppl2):S80-9.

13. Mravčík V, Strada L, Stolfa J, Bencko V, Groshkova T, Reimer J, Schulte B. Factors associated with uptake, adherence, and efficacy of hepatitis $\mathrm{C}$ treatment in people who inject drugs: a literature review. Patient Preference and Adherence 2013:7 1067-1075.

14. Butt AA, McGinnis KA, Skanderson and Justice AC. Hepatitis C treatment completion rates in routine clinical care. Liver Int. 2010;30(2):240-250.

14. Grebely J, Genoway KA, Raffa JD, Dhadwal G, Rajan T, Showler G et al. Factors associated with uptake of treatment for recent hepatitis $\mathrm{C}$ virus infection in a 
predominantly injecting drug user cohort: The ATAHC Study. Drug and Alcohol Dependence 2010;107:244-249.

16. Harris $M$ and Rhodes T. Hepatitis $C$ treatment access and uptake for people who inject drugs: a review mapping the role of social factors. Harm Reduction Journal

353 2013, 10:7 doi:10.1186/1477-7517-10-7.

18. Pearlman BL. Protease inhibitors for the treatment of chronic hepatitis C genotype-1 infection: the new standard of care. The Lancet Infect Dis.

17. Martin NK, Hickman M, Miners A, Hutchinson SJ, Taylor A and Vickermand P. Cost-effectiveness of $\mathrm{HCV}$ case-finding for people who inject drugs via dried blood spot testing in specialist addiction services and prisons. BMJ Open 2013;3:e003153. doi:10.1136/bmjopen-2013-003153.

362

363 19. Lange CM and Zeuzem S. Perspectives and challenges of interferon-free therapy 364 for chronic hepatitis C. J Hepatol 2013;58(3):583-592.

20. Afdhal NH, Zeuzem S, Schooley RT, Thomas DL, Ward JW, Litwin AH. The new paradigm of hepatitis $\mathrm{C}$ therapy: integration of oral therapies into best practices. Viral Hepat. 2013;20(11):745-760. doi: 10.1111/jvh.12173. 